\title{
PRIMING INTRAMODAL E INTERMODAL: UN ESTUDIO GUSTATIVO-VISUAL
}

\author{
Eugenia Razumiejczyk ${ }^{1}$, Guillermo Macbeth ${ }^{2}$ y J. Fernando Adrover ${ }^{3}$ \\ Consejo Nacional de Investigaciones Científicas y Técnicas (CONICET), Argentina \\ (RECiBIDO EL 03/12/2007, ACEPTADO El 30/05/2008)
}

\begin{abstract}
RESUMEN
El propósito de este estudio es evaluar el priming intramodal del gusto e intermodal entre el gusto y la visión. Los datos mostraron una facilitación en el reconocimiento de los estímulos estudiados en relación con los estímulos nuevos en el experimento de priming intramodal. En el experimento de priming intermodal, el reconocimiento de los estímulos estudiados no se diferenció significativamente del reconocimiento de los estímulos nuevos. En ambos estudios, los resultados mostraron que el tiempo de procesamiento requerido para los estímulos estudiados es menor que para los estímulos nuevos, y se observó una correlación inversa entre esta variable y el reconocimiento. Se concluye la presencia de priming intramodal del gusto e intermodal entre el gusto y la visión.
\end{abstract}

Palabras clave: Priming intramodal, Priming intermodal, gusto, visión.

\begin{abstract}
The aim of this study is to evaluate within and across priming between taste and vision. The data showed a facilitation in recognition of studied stimuli in relation to new stimuli in the experiment of within priming. In the experiments of within and across priming, the recognition of the studied stimuli did not differ significantly from the recognition of new stimuli. In both studies, the results showed that the processing time required to recognize studied stimuli were smaller than the time required for new stimuli. There was a negative correlation between processing time and recognition. The occurrence of within and across priming between taste and vision are consistent with the evidence of this studies.
\end{abstract}

Keywords: Within priming, Across priming, Taste, Vision.

1 Lic. en Psicología. Becaria Doctoral del Consejo Nacional de Investigaciones Científicas y Técnicas (CONICET). Argentina.

E-mail: eugeniaraz@fibertel.com.ar

2 Doctor en Psicología. Becario postdoctoral del Consejo Nacional de Investigaciones Científicas y Técnicas (CONICET). Argentina.

E-mail: guillermo.macbeth@mail.salvador.edu.ar

3 Lic. en Psicología. Universidad de Buenos Aires. Argentina.

E-mail: j.adrover@vaneduc.edu.ar 


\section{INTRODUCCIÓN}

La memoria implícita es recuperación no intencional de la experiencia previa que, sin que el sujeto sea consciente de que está experimentando un recuerdo, influencia su comportamiento actual (Tulving \& Schacter, 1990). El efecto de priming hace referencia a la influencia que tiene un estímulo en el rendimiento subsiguiente del sistema de procesamiento (Schacter, 1995; Schacter, Alapert, Savage, Rauch \& Albert 1996). El término priming es un concepto técnico de la psicología cognitiva que carece de una traducción precisa a la lengua española. El paradigma experimental en los estudios que intentan aportar evidencia empírica en relación al procesamiento implícito de la información señala dos fases en el experimento. En la fase de estudio, se presenta una serie de estímulos conocidos como estímulos estudiados. Posteriormente, se administra al participante una tarea distractora cuya función es operacionalizar el paso del tiempo. Luego, en la fase de prueba se presentan los estímulos estudiados junto con otros estímulos que no fueron presentados previamente; esto es, estímulos nuevos. El análisis de los datos obtenidos consiste, entonces, en comparar los resultados del procesamiento cognitivo en los estímulos estudiados y en los estímulos nuevos. Así, la medición de las variables dependientes mostrará una diferencia significativa entre estos estímulos a pesar del tiempo transcurrido.

El proceso cognitivo de la percepción involucra numerosos juicios de modo que producen una activación compleja de patrones sensoriales entre varias modalidades perceptivas (Frank, Van der Klaauw \& Schifferstein, 1993). La experiencia perceptiva del participante en las pruebas de memoria es multidimensional (Halpern, 2005; Rolls, 2004; Sekuler \& Blake, 2002; White \& Prescott, 2007). El estudio del priming intramodal o intermodal es pertinente desde el punto de vista de la psicología cognitiva dado que permite realizar inferencias sobre la naturaleza de las representaciones mentales que subyacen a la memoria implícita. La distinción entre estas pruebas implícitas implica diferentes manipulaciones experimentales (Cabeza, 1994). En el estudio del efecto de priming intramodal, los estímulos presentados en la fase de estudio y en la fase de prueba del experimento se encuentran en la misma modalidad perceptiva. Este fenómeno se expresa en pruebas indirectas en las que el procesamiento está determinado por las propiedades físicas de las claves del experimento, es un fenómeno presemántico que refleja la actividad del Sistema de Representación Perceptual (Tulving \& Schacter, 1990). Este sistema se caracteriza por ser de dominio específico de modo que está constituido por varios subsistemas con comunes propiedades y operaciones (Tulving \& Schacter, 1990). Así, además del subsistema de palabra en forma visual, del subsistema de descripción estructural y del subsistema de palabra en forma auditiva, la evidencia acumulada muestra que se incluye como subsistema de procesamiento implícito a la modalidad háptica (Ballesteros, Reales \& Manga, 1999), la olfativa (Koenig, Bourron \& Royet, 2000) y, posiblemente, también la gustativa (Razumiejczyk \& López Alonso, 2006; Razumiejczyk, Adrover, Macbeth \& López Alonso, 2007). Por el contrario, en el estudio del efecto de priming intermodal, la modalidad perceptiva de los estímulos presentados en la fase de estudio y en la fase de prueba es diferente (Ballesteros et al., 1999). El priming intermodal se manifiesta en pruebas indirectas en las que se requiere un procesamiento conceptual de los estímulos. Así, puede verse afectado por las operaciones de codificación semántica siendo apenas sensible a los cambios de las propiedades superficiales de la información. Refleja la actividad del Sistema Semántico de modo que el procesamiento 
de los estímulos y su recuperación implícita se encuentra en función de la organización semántica (Tulving \& Schacter, 1990).

\section{Pre-estudio}

El propósito es seleccionar un conjunto confiable de estímulos gustativos que resulten adecuados para poner a prueba las hipótesis correspondientes a los experimentos 1 y 2 vinculadas al efecto de priming intramodal del gusto y al efecto de priming intermodal entre el gusto y la visión. Se utilizan estímulos gustativos naturales en concordancia con su funcionalidad ecológica (Dhami, Hertwig \&Hoffrage, 2004; López Alonso, 2006; López Alonso, Razumiejczyk \& Macbeth, 2007). Los estímulos se presentan a los participantes en forma de papilla a temperatura ambiente. Esta forma de administración intenta prever que no se reconozca el objeto del gusto por su textura y temperatura y, a su vez, que los participantes puedan efectuar los movimientos naturales de la boca al comer. Así, se estudia el reconocimiento del gusto sin la participación de la percepción somatosensorial, tal como lo sugieren Cerf-Ducastel, Van De Moortele, Mac Leod, Le Bihan \& Faurion (2001), Berlucchi, Moro, Guerrini \& Aglioti (2004) y Razumiejczyk, Macbeth \& López Alonso (2007). Asimismo, se obstruyó la visión de modo que el color de los estímulos no influyera en su reconocimiento (Hoegg \& Alba, 2007). En un primer momento, se solicitó a un grupo de 10 sujetos que, por el período de dos semanas, registraran aquellas frutas y verduras que ingerían. A partir de la información recolectada, se seleccionaron aquellos alimentos que eran habitualmente ingeridos en la vida cotidiana, de modo que su administración en los posteriores experimentos respetara la relación ecológica entre el sujeto y su ambiente. Los estímulos gustativos seleccionados en esta etapa fueron plátano, pera, manzana, fresa, ananá (piña), durazno, calabaza, zanahoria, calabacín y brécol. La etiqueta empleada en este artículo para denominar los estímulos gustativos respeta las normas del Diccionario de la Real Academia Española (vigésima segunda edición). Se evaluó el reconocimiento de los estímulos gustativos seleccionados en la fase anterior. Participaron 30 sujetos argentinos universitarios cuya edad promedio resultó de 24,36 años $(d e=3,856$ años). La población femenina $(73,3 \%)$ predominó sobre la masculina $(26,7 \%)$. Los participantes fueron no fumadores y se les informó que no debían ingerir ningún alimento ni bebida que no fuera agua durante las tres horas previas al estudio. Se obstruyó la visión del participante con un antifaz mientras que el experimentador le presentaba cada uno de los diez estímulos gustativos que debía degustar y reconocer. Se informaba al participante que realizara una limpieza bucal ingiriendo unos sorbos de agua. Se consignaba que ponga agua en su boca, enjuague y trague antes de cada ensayo. Los alimentos fueron entregados en un orden aleatorio que se obtuvo mediante un programa estadístico para computadoras personales (Random Number Generator).

Los resultados mostraron que la probabilidad de acierto en el reconocimiento de los estímulos plátano, pera, manzana, fresa, ananá (piña), durazno, calabaza y calabacín fluctuó entre 0,40 y 0,80. El Alpha de Cronbach para el reconocimiento de estos estímulos fue de 0,686 . La probabilidad de acierto para los estímulos de zanahoria y brécol fue menor al 0,40 , así, se decidió excluirlos en los posteriores experimentos por ser demasiado difíciles de reconocer. Su no reconocimiento se debería, en parte, a la dificultad misma del estímulo más que a la manipulación de las variables independientes. 


\section{MÉTODO}

\section{Experimento 1}

El propósito de este experimento es evaluar el efecto de priming intramodal del gusto. La primera hipótesis experimental $H_{1}$ afirma que el reconocimiento $R$ es mayor para los estímulos gustativos estudiados $R E_{e}$ que para los estímulos nuevos $R E_{n}$. Formalmente, $H_{1}: R E_{e}>R E_{n}$. La hipótesis $H_{2}$ afirma que el tiempo de reacción $T$ es menor para los estímulos gustativos estudiados $T E_{e}$ que para los estímulos nuevos $T E_{n}$. Formalmente, $H_{2:} T E_{e}<T E_{n}$. La $H_{3}$ afirma que el reconocimiento $R$ y el tiempo de reacción $T$ de los estímulos estudiados $E_{e}$ se encuentran correlacionados inversamente. Formalmente, $\mathrm{r}_{R E e \text {, }}$ TEe $<0$. Las hipótesis $H_{1}, H_{2}$ y $H_{3}$ sugieren la presencia del efecto de priming gustativo desde las variables del reconocimiento y del tiempo de reacción.

Participantes. Participaron del experimento 33 sujetos argentinos universitarios cuya edad promedio resultó de 23,55 años ( $d e=6,06$ años). La población femenina $(69,7 \%)$ predominó sobre la masculina $(30,37 \%)$. Los participantes fueron no fumadores y se les informó que no debían ingerir ningún alimento ni bebida que no fuera agua durante las tres horas previas al experimento. Se estableció esta consigna durante el reclutamiento de los participantes que se realizó una semana antes del estudio. Al inicio del experimento, se verificó el cumplimiento de estas condiciones.

Procedimiento. Se entrenó a un grupo de seis experimentadores voluntarios quienes no conocieron el propósito del estudio hasta finalizada la toma de datos, de modo que funcionaron como experimentadores ciegos. Cada experimento fue realizado por dos experimentadores. La agrupación de los estímulos gustativos en estímulos estudiados y estímulos nuevos fue aleatoria y se obtuvo mediante un programa estadístico para computadoras personales (Random Number Generator). Los estímulos estudiados fueron pera, manzana, calabaza y durazno, mientras que los estímulos nuevos fueron fresa, ananá, plátano y calabacín. Sobre un escritorio se ubicaban ocho recipientes tapados, cada uno con un estímulo gustativo. A un costado se encontraba un cesto de residuos cuya función era la de contener los materiales ya utilizados. El experimentador 1 pedía el ingreso del participante y constataba que haya cumplido con los dos requisitos para el estudio, es decir, ser no fumador y no haber ingerido ningún alimento ni bebida que no fuera agua durante las últimas tres horas. Le solicitaba que tomara asiento frente al escritorio y explicaba el procedimiento de la fase de estudio del experimento enfatizando que la totalidad de los materiales utilizados eran descartables. El experimentador 1 pedía información acerca de su edad que el experimentador 2 completaba en el protocolo de respuestas junto con su sexo. Luego, el experimentador 1 entregaba al participante una servilleta de papel y un vaso descartable con agua recordándole que debía beber unos sorbos antes de cada ensayo. En la fase de estudio, el experimentador 1 presentaba al participante cada uno de los cuatro estímulos gustativos que debía degustar e informar si el gusto que percibía era salado o dulce. El experimentador 2 registraba estos datos en el protocolo de respuestas. Luego, el experimentador 1 arrojaba la cucharita utilizada para ese ensayo. Se informaba al participante que realizara una limpieza bucal ingiriendo unos sorbos de agua. Se consignaba que ponga agua en su boca, enjuague y trague. Los estímulos presentados en esta fase fueron pera, manzana, calabaza y durazno. A continuación, el experimentador 
1 entregaba al participante una tarea sencilla de papel y lápiz. Esta actividad funcionaba como tarea distractora. El tiempo para responder a las preguntas que contenían la tarea era de aproximadamente 20 minutos. Al finalizar la tarea, se proseguía con la fase de prueba del experimento. El experimentador 1 solicitaba al participante que bloqueara su visión con un antifaz. El procedimiento fue similar al de la fase de estudio, aunque aquí se entregaban los cuatro estímulos previos (pera, manzana, calabaza y durazno) más cuatro estímulos nuevos (fresa, ananá, plátano y calabacín). El participante debía reconocer el estímulo gustativo en el menor tiempo posible. Se tomó el tiempo en segundos desde el momento en que el estímulo era entregado en la boca del participante hasta que éste diera una respuesta. La tarea del experimentador 2 consistía en registrar los datos del estudio en el protocolo. Al finalizar el experimento, el experimentador 1 agradecía al participante la colaboración y lo acompañaba a la puerta de salida. Se arrojaban al cesto de residuos el vaso descartable y la servilleta de papel utilizados. Al concluir la jornada, los experimentadores arrojaban, además, la totalidad de los recipientes que contenían los estímulos gustativos. Los alimentos fueron entregados a cada participante en un orden aleatorio que se obtuvo mediante un programa estadístico para computadoras personales (Random Number Generator).

\section{RESULTADOS Y DISCUSIÓN (1)}

La $H_{1}$ resultó coherente con la evidencia experimental. La media del reconocimiento para los estímulos estudiados resultó de $R E_{e}=2,97$ ( $\left.d e=1,07\right)$, mientras que la media del reconocimiento para los estímulos nuevos fue de $R E_{n}=2,55(d e=1,12)$. Con el fin de comparar el reconocimiento de los estímulos estudiados y los estímulos nuevos se efectuó una prueba $t$ para muestras apareadas. La diferencia de medias resultó significativa $(t=$ $2,435 ; p=0,021)$. Así, los participantes mostraron un mejor reconocimiento cuando los estímulos gustativos fueron procesados durante la fase de estudio. La distribución de las variables fue compatible con la presunción de normalidad y homocedasticidad por las pruebas de Kolmogorov-Smirnov y Levene, respectivamente.

La hipótesis $H_{2}$ resultó coherente con la evidencia experimental. La media del tiempo de reacción para los estímulos estudiados resultó de $T E_{e}=32,64$ segundos $(d e=16,83$ segundos), mientras que la media del tiempo de reacción de los estímulos nuevos fue de $T E_{n}$ $=40,33$ segundos ( $d e=19,02$ segundos). Con el fin de comparar el tiempo de reacción de las respuestas de los participantes en los estímulos estudiados y en los estímulos nuevos se efectuó una prueba $t$ para muestras apareadas. Los resultados mostraron que el tiempo de reacción fue significativamente menor cuando los estímulos habían sido presentados en la fase de estudio $(t=-2,720 ; p=0,01)$. Las variables comparadas resultaron normales y homocedásticas según las pruebas de Kolmogorov-Smirnov y Levene, respectivamente.

La $H_{3}$ resultó coherente con la evidencia experimental. El reconocimiento de los estímulos estudiados $R E_{e}$ y el tiempo de reacción de los estímulos estudiados $T E_{e}$ se encontraron correlacionados inversamente $(r=-0,591 ; p<0,01)$. Así, los datos señalan que el reconocimiento de los estímulos estudiados en la tarea de memoria implícita requirió menos tiempo de procesamiento. 
El análisis conjunto de los resultados muestra que se efectuó un proceso de facilitación tanto en el reconocimiento, como en el tiempo de procesamiento de los estímulos estudiados en comparación con los estímulos nuevos. Estos datos sugieren que se produjo un efecto de priming gustativo en función del reconocimiento y del tiempo de reacción. Estos hallazgos se encuentran en concordancia con estudios previos (Henson, 2003; Razumiejczyk \& López Alonso, en prensa; Schott, Richardson-Klavehn, Henson, Becker, Heinze \& Düzel, 2006; Squire, Ojemann, Meizin, Petersen, Videen \& Raichle., 1992; Tulving \& Schacter, 1990; Wagner, Maril \& Schacter, 2000; Wig, Grafton, Demos \& Kelley, 2005). Así, luego de la exposición al estímulo, el procesamiento perceptual subsiguiente es más eficiente produciendo tiempos de reacción más rápidos.

\section{Experimento 2}

El propósito de este experimento es evaluar el efecto de priming intermodal entre el gusto y la visión. La primera hipótesis $H_{1}$ afirma que el reconocimiento $R$ de los estímulos gustativos estudiados $E_{e}$ y de los estímulos nuevos $E_{n}$ no se diferencian significativamente. Formalmente, $R E_{e} \approx R E_{n}$. La $H_{1}$ se justifica por la codificación semántica de los estímulos gustativos estudiados, de modo que se activarán en la memoria semántica determinadas representaciones abstractas y amodales. La hipótesis $H_{2}$ afirma que el tiempo de reacción $T$ de los estímulos gustativos estudiados $E_{e}$ es menor al de los estímulos nuevos $E_{n}$. Formalmente, $T E_{e}<T E_{n}$. La $H_{3}$ afirma que el reconocimiento $R$ y el tiempo de reacción $T$ de los estímulos estudiados $E_{e}$ se encuentran correlacionados inversamente. Formalmente, $r h o_{\text {REe, TEe }}<0$. Las hipótesis $\mathrm{H}_{2}$ y $\mathrm{H}_{3}$ sugieren la presencia de priming intermodal. Se espera que el tiempo de procesamiento en el reconocimiento de los estímulos estudiados sea menor cuando éstos han sido procesados en la fase de estudio del experimento.

Participantes. Participaron del experimento 33 sujetos argentinos universitarios cuya edad promedio resultó de 24,33 años ( $d e=5,337$ años). La población femenina $(78,8 \%)$ predominó sobre la masculina $(21,2 \%)$. El criterio de inclusión de la muestra fue el mismo que en el experimento 1.

Procedimiento. Se entrenó a un grupo de seis voluntarios quienes no conocieron el propósito del estudio hasta finalizada la toma de datos, de modo que funcionaron como experimentadores ciegos. La selección de los estímulos gustativos fue aleatoria y se obtuvo mediante un programa estadístico para computadoras personales (Random Number Generator). Los estímulos estudiados fueron manzana, calabacín y plátano. Los estímulos visuales estudiados fueron rojo (manzana), verde (calabacín) y amarillo (plátano). Los estímulos nuevos fueron marrón, violeta y naranja. Cada experimento fue realizado por dos experimentadores. Sobre un escritorio se ubicaban tres recipientes tapados, cada uno con un estímulo gustativo. A un costado se encontraba un cesto de residuos cuya función era la de contener los materiales ya utilizados. El experimentador 1 pedía el ingreso del participante y constataba que haya cumplido con los dos requisitos para el estudio, es decir, ser no fumador y no haber ingerido ningún alimento ni bebida que no fuera agua durante las últimas tres horas. Le solicitaba que tomara asiento frente al escritorio y explicaba el procedimiento de la fase de estudio del experimento enfatizando que la totalidad de los materiales utilizados eran descartables. El experimentador 1 pedía información acerca 
de su edad que el experimentador 2 completaba en el protocolo de respuestas junto con su sexo. Luego, el experimentador 1 entregaba al participante una servilleta de papel y un vaso descartable con agua recordándole que debía beber unos sorbos antes de cada ensayo. En la fase de estudio, el experimentador 1 presentaba al participante cada uno de los tres estímulos gustativos que debía degustar y realizar una codificación semántica. La consigna de esta fase del experimento consistía en preguntar al participante: "Si en un restaurante te sirven una comida que tiene el ingrediente que probaste, ese ingrediente ¿formaría parte del plato principal o del postre? Nombra un plato principal/postre en dónde se utilice ese ingrediente". Luego, el experimentador 1 arrojaba la cucharita utilizada para ese ensayo. El experimentador 2 registraba estos datos en el protocolo de respuestas. Los estímulos gustativos presentados en esta fase fueron manzana, calabacín y plátano. Se informaba al participante que realizara una limpieza bucal ingiriendo unos sorbos de agua. Se consignaba que ponga agua en su boca, enjuague y trague. A continuación, el experimentador 1 entregaba al participante una tarea sencilla de papel y lápiz. Esta actividad funcionaba como tarea distractora. El tiempo para responder a las preguntas que contenía la tarea era de aproximadamente 20 minutos. Al finalizar la tarea, se proseguía con la fase de prueba. Se presentaba en la pantalla de una computadora seis colores de modo sucesivo. El participante debía informar lo más rápido posible aquello que se le viniera a la mente a partir del estímulo visual observado. Se tomó el tiempo en segundos desde el momento en que el estímulo era presentado en la pantalla hasta que el participante diera una respuesta. La tarea del experimentador 2 consistía en registrar los datos del estudio en el protocolo. Al finalizar el experimento, el experimentador 1 agradecía al participante la colaboración y lo acompañaba a la puerta de salida. Se arrojaban al cesto de residuos el vaso descartable y la servilleta de papel utilizados. Al concluir la jornada, los experimentadores arrojaban, además, la totalidad de los recipientes que contenían los estímulos gustativos. Los alimentos y los estímulos visuales fueron presentados a cada participante en un orden aleatorio que se obtuvo mediante un programa estadístico para computadoras personales (Random Number Generator).

\section{RESULTADOS Y DISCUSIÓN (2)}

La hipótesis $H_{1}$ resultó coherente con la evidencia experimental. La media del reconocimiento para los estímulos estudiados resultó de $R E_{e}=1,30(d e=1,15)$ con una mediana de 1 . La media del reconocimiento para los estímulos nuevos resultó de $R E_{n}=1,21(d e=1,08)$ con una mediana de 1 . La distribución de estas variables fue compatible con la presunción homocedasticidad por la prueba de Levene, e incompatible con la presunción de normalidad por la prueba de Kolmogorov-Smirnov. Con el fin de comparar el reconocimiento de los estímulos estudiados y de los estímulos nuevos, se efectuó la prueba de Wilcoxon cuyo resultado mostró que estas variables no se diferenciaron significativamente $(p=0,527)$. Estos resultados señalan que luego del procesamiento semántico de los estímulos gustativos efectuado en la fase de estudio del experimento, los estímulos visuales administrados en la fase de prueba produjeron tanto una facilitación implícita en el reconocimiento de los estímulos gustativos estudiados, así como una facilitación de otros estímulos gustativos no presentados previamente. De este modo, al administrar colores nuevos que no se relacionaban con los colores naturales de los estímulos estudiados (manzana = rojo; 
plátano $=$ amarillo; calabacín $=$ verde), los participantes tendieron a responder con diversas frutas y verduras. Por ejemplo, frente al color violeta se encontraron respuestas tales como remolacha, uva, lechuga, frambuesa y ciruela; frente al color naranja, durazno, naranja, mandarina y calabaza; y frente al color marrón, chocolate, puerro, cebolla y calabaza. Estos datos sugieren que, luego de la codificación semántica de los estímulos gustativos, se activó en la memoria semántica determinadas representaciones abstractas y amodales relativas a la categoría de frutas y verduras que produjo una facilitación de las respuestas en la fase de prueba del experimento. De este modo, no se produjo una ventaja en la facilitación del reconocimiento de los estímulos estudiados en relación a los estímulos visuales nuevos. Estos resultados permiten explicar investigaciones anteriores en las que no se logró encontrar evidencia empírica de priming intermodal en otras modalidades perceptivas. Así, Koenig et al. (2000) no lograron mostrar el efecto de priming intermodal entre el olfato y la visión, debido a que no se logró la activación semántica de los estímulos olfativos presentados en la fase de estudio. Estos datos, junto con los resultados presentados en este estudio, se encuentran en concordancia con la idea de que el priming intermodal involucra componentes representacionales abstractos (Curran, Schacter \& Galluccin, 1999; Richardson-Klavehn \& Gardiner, 1996) y amodales accesibles a distintos sistemas perceptivos (Ballesteros \& García Rodríguez, 2000).

La hipótesis $\mathrm{H}_{2}$ resultó coherente con la evidencia experimental. La media del tiempo de reacción para los estímulos estudiados resultó de $T E_{e}=12,55$ segundos $(d e=4,41$ segundos), mientras que la media del tiempo de reacción de los estímulos nuevos fue de $T E_{n}=19,18$ segundos ( $d e=9,14$ segundos). Con el fin de comparar el tiempo de reacción de las respuestas de los participantes en los estímulos estudiados y en los estímulos nuevos, se efectuó una prueba $t$ para muestras apareadas. Los resultados mostraron la presencia de una diferencia significativa $(t=4,46 ; p<0,01)$. Así, el tiempo de reacción fue significativamente menor cuando los estímulos habían sido presentados en la fase de estudio. Las variables comparadas resultaron normales y homocedásticas según las pruebas de Kolmogorov-Smirnov y Levene, respectivamente.

La $H_{3}$ resultó coherente con la evidencia experimental. Se efectuó la correlación rho de Spearman que arrojó la presencia de una correlación negativa entre el reconocimiento de los estímulos estudiados $R E_{e}$ y el tiempo de reacción de los estímulos estudiados $T E_{e}$ (rho $=-0,654 ; p<0,01)$.

El análisis conjunto de los resultados muestra que, si bien el tiempo de procesamiento resultó menor cuando los estímulos habían sido presentados en la fase de estudio en comparación con los estímulos visuales nuevos, el reconocimiento no se diferenció significativamente. Estos datos sugieren que, luego de la activación de representaciones abstractas y amodales relativas a frutas y verduras en la memoria semántica, los participantes respondieron a los estímulos visuales a partir de esas representaciones activadas. Los resultados muestran la presencia de priming intermodal entre el gusto y la visión dado que el tiempo de procesamiento en el reconocimiento de los estímulos es menor cuando éstos han sido procesados en la fase de estudio del experimento. 


\section{DISCUSIÓN GENERAL Y CONCLUSIONES}

Se ha realizado el experimento 1 cuyo objetivo fue estudiar el efecto priming intramodal del gusto. Los resultados mostraron que el reconocimiento de los estímulos gustativos estudiados resultó mayor que el de los estímulos nuevos, necesitando menos tiempo para su procesamiento. Así, se produjo una facilitación tanto en el reconocimiento, como en el tiempo de reacción para los estímulos gustativos estudiados. A su vez, el reconocimiento de los estímulos estudiados y el tiempo de reacción se encontraron correlacionados inversamente. El análisis conjunto de estos datos sugiere que se ha producido un efecto de priming de repetición en la modalidad sensorio-perceptiva del gusto. Resulta relevante sugerir la presencia de un subsistema específico encargado del procesamiento implícito de la información gustativa en el Sistema de Representación Perceptual. Se ha realizado el experimento 2 cuyo objetivo fue estudiar el efecto priming intermodal entre el gusto y la visión. Los resultados mostraron que el reconocimiento de los estímulos estudiados y de los estímulos nuevos no se diferenció significativamente. Estos datos muestran que, luego del procesamiento semántico de los estímulos gustativos en la fase de estudio del experimento, los estímulos visuales administrados en la fase de prueba produjeron tanto una facilitación implícita en el reconocimiento de los estímulos gustativos estudiados, como una facilitación de otros estímulos gustativos no presentados previamente. Luego de la codificación semántica de los estímulos gustativos, se activó en la memoria semántica la categoría de frutas y verduras que produjo una facilitación de respuestas relativas a estos conceptos en la fase de prueba del experimento. De este modo, no se produjo una ventaja en la facilitación del reconocimiento de los estímulos estudiados en relación a los estímulos visuales nuevos. El tiempo de reacción para los estímulos estudiados resultó significativamente menor que para los estímulos nuevos. A su vez, el reconocimiento de los estímulos estudiados y el tiempo de reacción se encontraron inversamente correlacionados. En este sentido, los resultados muestran la presencia de priming intermodal entre el gusto y la visión. Así, se concluye que el priming intermodal involucra componentes representacionales abstractos y amodales accesibles a distintos sistemas perceptivos, esto es, el gusto y la visión.

Los hallazgos de este estudio recomiendan investigar la vinculación de las representaciones gustativas y visuales en la percepción del sabor (Hoegg \& Alba, 2007).

\section{REFERENCIAS BIBLIOGRÁFICAS}

1. Ballesteros, S., Reales, J. y Manga, D. (1999). Memoria implícita y memoria explícita intramodal e intermodal: influencia de las modalidades elegidas y del tipo de estímulos. Psicothema 11(4), 831-851.

2. Ballesteros, S. y García Rodríguez, B. (2000). Procesos psicológicos básicos. España: Editorial Universitas.

3. Berlucchi, G.; Moro, V.; Guerrini, C. \& Aglioti, S. M. (2004). Dissociation between taste and tactile extinction en the tongue after right brain damage. Neuropsychology 42, 1007-1016. 
4. Cabeza, R. (1994). A dissociation between two implicit conceptual tests supports the distinction between types of conceptual processing. Psychonomic Bulletin and Review 1(4), 505-508.

5. Cerf-Duscatel, B.; Van De Moortele, P. F.; Mac Leod, P.; Le Bihan, D. \& Faurion, A. (2001). Interaction of gustatory and lingual somatosensory perceptions at the cortical level in the human: a functional magnetic resonante imaging study. Chemical Senses 26, 371-383.

6. Curran, T.; Schacter, D.L. \& Galluccin, L. (1999). Cross modal priming and explicit memory in patients with verbal production deficits. Brain \& Cognition 39, 133-146.

7. Dhami, M.K.; Hertwig, R. \& Hoffrage, U. (2004). The role of representative design in an ecological approach to cognition. Psychological Bulletin 6, 959-988.

8. Frank, R.A.; Van der Klaauw, N.J. \& Schifferstein, H.N.J. (1993). Both perceptual and conceptual factors influence taste-odor and taste-taste interactions. Perception \& Psychophisics, 54(3), 343-354.

9. Halpern, B.P. (2005). Temporal characteristics of human taste judgements as calibrations for gustatory event-related potentials and gustatory magnetoencephalographs. Chemical Senses 30, 228-234.

10. Henson, R. N. (2003). Neuroimaging studies of priming. Neurobiology 70, 53-81.

11. Hoegg, J. \& Alba, J.W. (2007). Taste perception: more than meets the tongue. Journal of Consumer Research 33(4), 490-498.

12. Koenig, O., Bourron, G. \& Royet, J.P. (2000). Evidence for separate perceptive and semantic memories for odors: a priming experiment. Chemical Senses 25, 703708 .

13. López Alonso, A.O. (2006). El carácter ecológico de la percepción gestáltica. Memorias de las XIII Jornadas de Investigación de la Facultad de Psicología, tomo III, 440-441. Buenos Aires: Universidad de Buenos Aires.

14. López Alonso, A.O., Razumiejczyk, E. y Macbeth, G. (2007). La necesidad de un enfoque ecológico en la psicología cognitiva y en las ciencias sociales. Memorias de las XIV Jornadas de Investigación de la Facultad de Psicología, tomo III, 471-473. Buenos Aires: Universidad de Buenos Aires.

15. Razumiejczyk, E. y López Alonso, A.O. (2006). Disociaciones funcionales de la memoria y sustratos neuroanatómicos. Psico Logos 16, 35-50.

16. Razumiejczyk, E. y López Alonso, A.O. (en prensa). La variable de los niveles de procesamiento no tiene incidencia en el efecto de priming gustativo. Acta Psiquiátrica y Psicológica de América Latina.

17. Razumiejczyk, E., Macbeth, G. y López Alonso, A.O. (2007). La lateralización del sentido del gusto y sus evidencias neurofisiológicas. Investigaciones en Psicología 12(1), 93-103. 
18. Razumiejczyk, E., Adrover, J.F., Macbeth, G. y López Alonso, A.O. (2007). Evidencia neurofuncional de la disociación entre la memoria explícita e implícita. Acta Psiquiátrica y Psicológica de América Latina 53, 23-33.

19. Richardson-Klavehn, A. \& Gardiner, J.M. (1996). Cross-modality priming in ítem completion reflects conscious memory, but not voluntary memory. Psychonomic Bulletin \& Review 3(2), 238-244.

20. Rolls, E. T. (2004). The functions of the orbitofrontal cortex. Brain and Cognition 55, 11-29.

21. Schacter, D.L. (1995). Priming and multiple memory systems: perceptual mechanisms of implicit memory. En D.L. Schacter \& E. Tulving (Eds.), Memory Systems 1994 (pp. 233-268). Massachusetts: MIT Press.

22. Schacter, D. L., Alapert, N. M., Savage, C. R., Rauch, S. L. \& Albert, M. S. (1996). Conscious recollection and the human hippocampal formation: Evidence from positron emission tomography. Proceedings of the National Academy of Sciences 93, 321-325.

23. Schott, B., Richarson-Klavehn, A., Henson, R. N. A., Becker, C., Heinze, H. J. \& Düzel, E. (2006). Neuroanatomical dissociation of encoding processes related to primig and explicit memory. The Journal of Neuroscience 26(3), 792-800.

24. Sekuler, R. \& Blake, R. (2002). Perception. New York: McGraw Hill.

25. Squire, L.R., Ojemann, J.G., Meizin, F.M., Petersen, S.E., Videen, T.O. \& Raichle, M.E. (1992). Activation of the hippocampus in normal humans: a functional anatomical study of memory. Proceedings of the National Academy of Sciences 89, 1837-1841.

26. Tulving, E. \& Schacter, D. L. (1990). Priming and human memory systems. Science 247, 301-306.

27. Wagner, A. D., Maril, A. \& Schacter, D. L. (2000). Interactions between forms of memory: when priming hinders new episodic learning. Journal of Cognitive Neuroscience 12, 52-60.

28. White, T.L. \& Prescott, J. (2007). Chemosensory cross-modal stroop effects: congruent odors facilitate taste identification. Chemical Senses, 32(4), 337-341.

29. Wig, G. S., Grafton, S. T., Demos, K. E \& Kelley, W. M. (2005). Reductions in neural activity underlie behavioral components of repetition priming. Nature Neuroscience 8, 1228-1233. 